\title{
A Novel Control Plane Model of Extensible Routers ${ }^{\star}$
}

\author{
Kun $\mathrm{Wu}$, Jianping $\mathrm{Wu}$, and $\mathrm{Ke} \mathrm{Xu}$ \\ Department of Computer Science and Technology, \\ Tsinghua University, Beijing 100084, P.R. China \\ \{kunwu, xuke\}@csnet1.cs.tsinghua.edu.cn, \\ jianping@cernet.edu.cn
}

\begin{abstract}
The extensible router is the next generation router architecture. This paper studies the extensibility of the router control plane, which contains complicated modules such as routing protocol processing, router information management, etc. It is difficult to decide when to extending the control plane, and how many nodes are needed. Moreover, the module dispatching configurations is another issues. This paper describes the extensible architecture of the router system and the control plane. It defines three variables to evaluate the extensibility. And a twostep extensible model is provided to achieve the maximum extensibility under these evaluations. Finally, the experiments show that the model is correct and efficient.
\end{abstract}

\section{Introduction}

\subsection{Motivation}

The next generation router will concentrate in the extensible architecture [1] [2] 3 . The single chassis router, which has been widely used in the conventional router system, cannot catch up with the Internet explosion [4. A fully extensible structure is borrowed from computer clusters [5], which can help in meeting the expanding requirements of the future Internet.

In the recent years, research on the scalability in the data plane has grown enormously. However, very few solutions can be found by far on how to make a control plane extensible enough, which is as critical as that of the the data plane. While the system is becoming larger and larger by clustering more small nodes, the routing protocol processing and other service routines can cause bottlenecks in both of the processing power and the memory storage.

\subsection{Previous Work}

The router extensibility is an interesting topic. Some demos or products under the extensible architectures have been released. The systems can be extended

\footnotetext{
* This work was supported by the National Key Fundamental Research Plan (973) of China (No 2003CB314801).
} 
to multiple chassis with specific inter-connecting networks. Many manufactures announced their systems, such as the CRS from CISCO, T640 from Juniper and Avici's TSR system. The interconnecting styles embedded are very different. CRS uses the multi-stage inter-connecting network. And, TSR employs a 3D-torus architecture. However, the majority research and improvement are located in the extensibility on the data plane. Very few evolutions can be found on the control plane, which can not keep up with the extension on the date plane.

Nevertheless, some research groups are active on the extensible control plane, especially to provide an extensible router software platform. The Scout [1] is an configurable operating system, which can provide the functional extensibility. Click [6] is a module-oriented router model, which is composed by simple elements. Router Plugins [7] is also a way to extend the router functions by import new plugins onto the processing path. However, all of these models are mainly focusing on the functional extensibility, other than the structure extending. Zebra is an excellent module-based routing software platform. It is well designed and implemented under a structural manner. It can contribute much on the extensible router system although it is not extensible itself. The XORP [8] is an active project on providing a flexible and extensible software router platform for network reach. It provides a flexible well-defined structure. However it is not focus on the performance-driven extensibility.

In this paper, we analyze the evolution of the modern router architecture, and provide a feasible model for extensible router systems. Section 2 give a overview of the architecture of router and the control plane. Section 3 describes the extensibility requirements and analyze the key issues. Section 4 presents the extensible model in details. Section 5 evaluates the performance of the model. Section[6 gives a conclusion of the paper.

\section{Architecture Overview}

In this section, we will describe why an extensible model is necessary, and what the structure it looks like. Fig1 depicts the conceptual extensible router architecture. The forward entities are the components located in the data plane. They are connected by the interconnecting network. This is beyond this paper's discussion, which focus on the control plane.

As shown in Fig. 1, the control entities are the control part of each node. The system running control and routing processing are located in them, which constitute the control plane. The control entities are connected together by a communication network. This communication network can be implemented in different ways, such as a switch, or even a virtual network provided by the data plane. The main problem now is how to distribute the processing modules to different control entities in the control plane.

The control plane architecture is shown in Fig. 2. The concrete processes on each control entity are represented as modules, such as OSPF processing. The lines in Fig. 2 represent the relationships between each pair of nodes. 


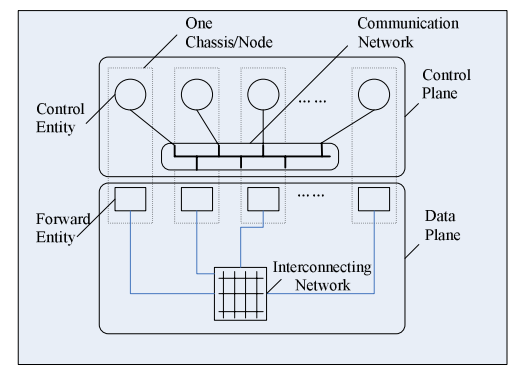

Fig. 1. Extensible Router Architecture

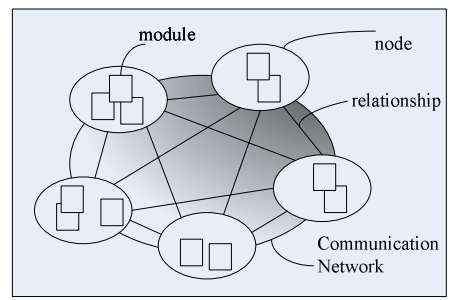

Fig. 2. Control Plane Architecture

\section{$3 \quad$ Extensibility Requirements}

Benefited from the optical technology improvements 9 10, the interface speed and density reach to an extreme high level. The core router will provide carrier class high availability to achieve non-stop forwarding [11. Moore's Law 12 shows that the computer performance would double every 18.6 months. But the data traffic in Internet has grown with a tremendous speed 13 [14, which is approximately doubling each year. It is not possible to support the traffic and processing in one box.

One of the key issues for extensibility is to limit the cost increasing during the extending. The overall cost is denoted as $C(n)$ for a $n$-nodes router. The effect function is $E(n)$, which is a integrated measurement for that the extension can benefit. We define the derivative $\frac{\Delta C(n)}{\Delta n}$ as the Extending Cost Rate (ECR). The $\frac{\Delta E(n)}{\Delta n}$ shows the efficiency, which is defined as the Extending Efficiency Rate (EER). The requirements for the extensibility is to minimize the ECR, and maximize the EER respectively. Moreover, we define Extending Balance Level (EBL) to reflect the balancing result, which can be measured by the geometric mean for the utilities on each node.

Under the current philosophy of parallel computing, it is impossible to reduce ECR to a level lower than zero. We define the inherent cost for one problem, i.e. routing processing in router, as $\hat{C}$. In the extended system with $n$ nodes, $\hat{C}$ can be distributed as $\hat{c_{i}}$, where $i=0,1, \cdots, n-1$. The communications among 
these nodes can introduce overheads, including the extra temporary memory, the inter-process communication, the scheduling, etc. The overhead on node $j$ caused by information issued by node $i$ is presented as $o_{i j}$. These overheads will add to the overall cost. We denote the relationship between node $i$ and node $j$ as $r_{i j}$, and the $\mathbf{R}_{i \times j}$ is the relation matrix. The sets of overheads and relation values are $\mathbb{O}$ and $\mathbb{R}$. A non-decreasing function $\mathscr{F}\left(r_{i j}\right): \mathbb{R} \mapsto \mathbb{O}$ can be given to convert the relationship to overhead. That is $o_{i j}=\mathscr{F}\left(r_{i j}\right)$. According to the information theory, the information from node $i$ to $j$ cannot be negative, $r_{i j} \geq 0$. And, there will be no overhead on node $j$ caused directly by node $i$, if node $j$ is not depended directly on node $i$. Then we have $\mathscr{F}(0)=0$. So for $\forall i, j=1,2, \cdots, n$, we have $o_{i j}=\mathscr{F}\left(r_{i j}\right) \geq \mathscr{F}(0)=0$. That is $o_{i j} \geq 0$. While being extended to $n$ nodes, the total cost is the accumulate cost and overhead on all the $n$ nodes. $C_{\text {all }}(n)=\Sigma c_{i}+\Sigma \Sigma o_{i j}=$ $\hat{C}+\Sigma \Sigma \mathscr{F}\left(r_{i j}\right)$. Then we have $\frac{\Delta C(n)}{\Delta n}=\frac{C_{\text {all }}(n)-C_{\text {all }}(n-1)}{n-(n-1)}=\Delta \Sigma \Sigma \mathscr{F}\left(r_{(}(i j)\right)$. It shows that the potential extensibility is controlled by the relationship matrix and the convert function. Let $\mathscr{G}(n)=\Sigma \Sigma \mathscr{F}\left(r_{i j}\right)$ be the integrated overhead for a $n$-nodes system under certain distributed scheme. Hence, the ECR can be written as

$$
\operatorname{ECR}(n)=\Delta \mathscr{G}(n)=\Delta \sum_{i=1}^{n} \sum_{j=1}^{n} \mathscr{F}\left(r_{i j}\right)
$$

For node $i$, we write the resources it can provided as $a_{i}$. The total resources in the extensible system will be $A(n)=\sum_{i=1}^{n} a(i)$. After cutting off the overhead, we get the available resources $A(n)-\mathscr{G}(n)$. Therefore, the EER can be written as

$$
\operatorname{EER}(n)=a_{n}-\Delta \mathscr{G}(n)=a_{n}-\operatorname{ECR}(n)
$$

And, by definition, we can write the extending balance level as

$$
E B L(n)=\left(\prod_{i=1}^{n} \frac{c_{i}}{a_{i}}\right)^{\frac{1}{n}}
$$

From these equations, we can point out that the extending result is dominated by the correlation between each pair of the nodes, the contribution of the relation to the cost, the capability of the new participant node, and the system-wise balance level.

\section{Extensibility Model}

Consider the situation that the information dependence of the tasks, which are separated onto the new coming node against those on the rest nodes, increase to a large value, so that the overhead will overwhelm the capacity. In the router systems, many high cost tasks are also too tightly coupled to be separated. At some point when the the overhead for dispatching a new module is greater than the incremental capacity, the extending upper-bound is reached. By the 
definitions, we can calculate the extension limitation for a system by the ECR and EER, so that the system can still be extended to more nodes. But it is not enough. Because it has no senses to assign a trivial module to a new node, although it will contribute to the ECR and EER. The balance level (EBL) decides which and how many modules should be dispatched. So, the EBL is used to evaluate if an extension scheme is good or bad while the node number is given.

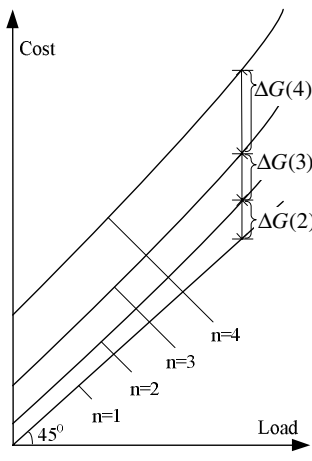

(a) Total Cost

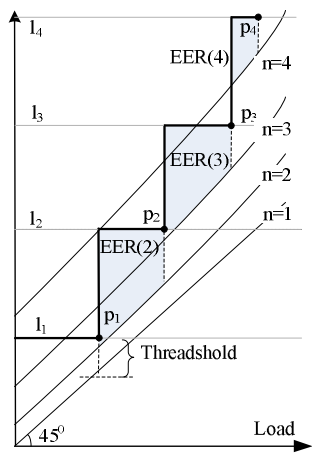

(b) Extending Path

Fig. 3. Extending Path

The extensibility model contains two parts. One is to decide how many nodes are needed for the given system load. The other is to assign the modules to these separated nodes.

The first step is depicted in Fig. 3. The left fig shows the cost increasing with the load under different amount of nodes. The number of nodes is denoted as $n$. $n=1$ means the single chassis system.

The function in Equation 1 is a converter from information to overhead. $\mathscr{F}\left(r_{i j}\right)$ is the overhead on node $j$ caused by the information issued by node $i$. While considering a real router system employ a multi-tasks operating system (OS), the job scheduling, bus arbitration, and buffer management can introduce the side effect of OS overhead. Long term measurement can show us that the processing overhead at node $j$, with the attached management overhead is counted in, can increase faster than the increment of information from $i$ to $j$. Hence, the conversion function $\mathscr{F}\left(r_{i j}\right)$ is roughly a convex function. This is why the curve with a larger node number is above that with a smaller one. And the space between every two adjacent curves has the same trend.

While the total node capacity is considered, we will have the relationship between system resource and overall cost along with variety of the load. This is illustrated in right part of Fig. 3. The horizontal lines from $l_{1}$ to $l_{4}$ is the capabilities with node number $n=1,2,3,4$. Each horizontal line is a resource level the system can provide. The space from the line to the corresponding cost curve describes the resource available. This space reduces with the load increasing. A threshold is introduced here to preserve some resources for the 
current configuration. While the threshold is reached, a decision must make if it will transfer to higher resource level. The decision points are shown as $p_{1}, p_{2}, p_{3}, p_{4}$. And, for each value of $n$, we will have the EER as the shadow areas.

If we connect each decision point and the next horizontal lines, which is depicted as the bold line in the right figure, a decision border is given. We name it as the Extending Path. The area above the path is all the permitted conditions. And the area below can be resource exhausted-prone situations. From this figure, we can decide how many nodes needed, and what efficiency the extension can achieve.

Now, the second step is to give a suitable modules distributing configuration so that the EBL is maximized. For simplicity, we will inspect the situation in a simple system, which has the typical modules as following.

- RTMGT: route management processing, i.e. forwarding table maintaining.

- OSPF: OSPF protocol processing

- BGP: BGP protocol processing

- TRANS: transport layer support computing, i.e. TCP/UDP/IP.

- MGMT: system management processing, i.e. MIB collecting.

And the information dependencies among them are listed below:

- MIB: Management Information Base.

- $R T_{B G P}$ : BGP routing table changes.

- $R T_{O S P F}$ : OSPF routing table changes.

- UPD $D_{B G P}$ : BGP update information.

- UPD $D_{O S P F}$ : OSPF update information.

We can sort the information dependencies in a more general sense. $M I B \gg \Delta R T_{B G P}>\Delta R T_{O S P F} \gg \Delta U P D_{B G P}>\Delta U P D_{O S P F}$

So, we can arrange the dependencies into the following table.

Table 1. Information Dependencies

\begin{tabular}{l|c|c|c|c|c}
\hline Source & $R T M G T$ & $O S P F$ & $B G P$ & $T R A N S$ & $M G M T$ \\
\hline$R T M G T$ & 0 & $\Delta R T_{B G P}$ & $\Delta R T_{O S P F}$ & $\varepsilon$ & $M I B$ \\
$O S P F$ & $\Delta R T_{O S P F}$ & 0 & 0 & $\Delta U P D_{O S P F}$ & $M I B$ \\
$B G P$ & $\Delta R T_{B G P}$ & 0 & 0 & $\Delta U P D_{B G P}$ & $M I B$ \\
$T R A N S$ & $\varepsilon$ & $\Delta U P D_{O S P F}$ & $\Delta U P D_{B G P}$ & 0 & $M I B$ \\
$M G M T$ & $\varepsilon$ & $\varepsilon$ & $\varepsilon$ & $\varepsilon$ & 0 \\
\hline
\end{tabular}

The source of the information flow is list in the first column. Each one of the rest columns is the information dependencies to the first item in the column. A small value $\varepsilon$ is introduced for the dependencies that can be ignored. From the table we can classify the modules into three levels. The Level $I$ is the closely associated module, i.e. the $M G M T$, is tightly coupled with every other modules. 
The Level $I I$ is the partly coupled module, such as $O S P F$ or BGP, which is much depend on some of the other partly coupled modules. The Level III is the weakly coupled service module, i.e. $T R A N S$, which depends loosely on other modules.

To balance the load on each node, we have the following guidelines for modules dispatching schemes.

1. Distribute Level I modules evenly to each node.

2. Distribute Level II modules in the descent order.

3. Distribute Level III modules with the corresponding modules in Level II.

For a router system, the task granularity cannot small enough to achieve a fully balanced configuration, because many resource-consuming tasks are composed by modules tightly-coupled. However, the guidelines can achieve the maximum EBL under this condition.

\section{$5 \quad$ Model Evaluations}

We simulate the 2-step extensible model under one PC. Each router node is implemented as a process, and the modules are programmed as resourcesconsuming threads. The router system is abstracted as a typical router system containing the modules BGP, OSPF, route management, Transport layer and management routines. The information dependencies are assigned as the dynamic traffics among the modules under the same manner as Table 1] The results are shown in Fig. 4 .

The experiment for extending decision points shows that the simulating results are almost the same as the decision path in Fig. 3. except that the simulating decision points come some earlier before the calculating. That's an error introduced by the dynamic randomness of the modules.

The experiment for the module dispatching configuration shows that the system load is balanced to some extend. The balance level is not far beyond the theoretical lower bound.
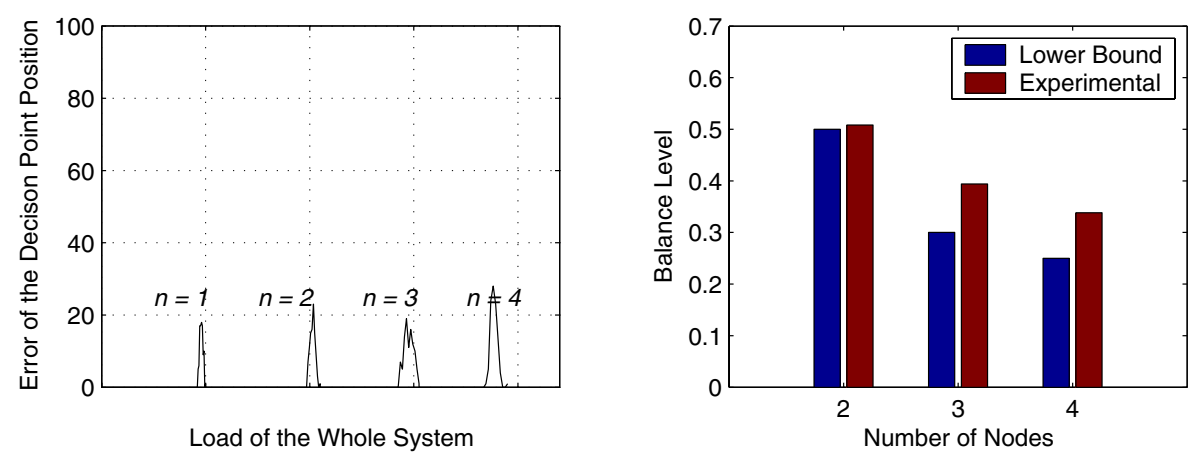

Fig. 4. Experiment Results 


\section{Conclusion}

The extensible router is the next generation core router architecture. This paper focuses on the extensibility of the control plane. The control plane structure is deeply inspected. EER, EFR, and EBL are given to evaluate the extension schemes. We present a two-steps extensible model in this paper. The first step is to decide how many nodes are needed for the extension and when to extend. The next step contains a list of rules to find out a modules configuration scheme so that the extension is balanced.

\section{References}

1. S.Karlin, L.Peterson. "VERA: An extensible router architecture", Computer Networks, Vol. 38, Issue 3 (February 2002).

2. Lukas Ruf, Ralph Keller, Bernhard Plattner, "A Scalable High-performance Router Platform Supporting Dynamic Service Extensibility On Network and Host Processors", ICPS'04, 2004.

3. H.J.Chao, "Next Generation Routers", Proceedings of the IEEE, vol.90, no.9, Sep. 2002, pp.1518-1558.

4. H.Jonathan Chao, Kung-li Deng, Zhigang Jing, "A Petabit Photonic Packet Switch $\left(\mathrm{P}^{3} \mathrm{~S}\right)$ ", IEEE INFOCOMM'03, San Francisco, April 1-3, 2003.

5. Buyya, R. (ed.), "High Performance Cluster Computing: Architectures and Systems", Volume 1 and 2, Prentice Hall PTR, NJ, USA, 1999.

6. E. Kohler, R. Morris, B. Chen, J. Jonnotti, M. F. Kaashoek, "The Click Modular Router", ACM Transactions on Computer Systems 18(3), Aug. 2000.

7. D Decasper, Z Dittia, G Parulkar, B Plattner, "Router Plugins: A Software Architecture for Next-Generation Routers" ACM Transactions on Networking, 2000.

8. M Handley, O Hodson, E Kohler, "XORP: An open platform for network research", Proceedings of HotNets-I Workshop, October, 2002.

9. F.Masetti, D.Zriny, etc, "Design and Implementation of a Multi-Terabit Optical Burst/Packet Router Prototype", OFC'2002, 2002.

10. Yang Chen, Chunming Qiao, Xiang Yu, "Optical Burst Switching(OBS): A New Area in Optical Networking Research", IEEE Network, 2004.

11. Avici Inc. "Non-Stop Routing Technology", white paper, 2002.

12. Gordon E. Moore, "Cramming More Components Onto Integrated Circuits", Electronics, April 19, 1965.

13. Roberts, L.G., "Beyond Moore's law: Internet growth trends", Computer Vol.33, Jan 2000.

14. K. G. Coffman and A. M. Odlyzko, "Internet growth: Is there a 'Moores Law' for data traffic?", in Handbook of Massive Data Sets. Norwell, MA: Kluwer, 2001 